\title{
Inverse relationship between obesity and FTO gene expression in visceral adipose tissue in humans
}

\author{
N. Klöting • D. Schleinitz • K. Ruschke • J. Berndt • \\ M. Fasshauer • A. Tönjes • M. R. Schön • P. Kovacs • \\ M. Stumvoll • M. Blüher
}

Received: 1 October 2007 / Accepted: 21 December 2007 / Published online: 5 February 2008

(C) Springer-Verlag 2008

\begin{abstract}
Aims/hypothesis Recently, FTO was identified as a candidate gene contributing to both childhood and severe adult obesity. We tested the hypothesis that mRNA expression of FTO and/or of the neighbouring RPGRIPIL in adipose tissue correlates with measures of obesity and fat distribution. We also investigated whether the FTO obesity risk alleles might explain variability in FTO and RPGRIPIL mRNA expression.

Methods In paired samples of visceral and subcutaneous adipose tissue from 55 lean and obese participants, we investigated whether FTO and RPGRIPIL mRNA expression is fat depot-specific, altered in obesity and related to measures of fat accumulation, insulin sensitivity and glucose metabolism. All participants were genotyped for the obesity-associated rs8050136 FTO variant.
\end{abstract}

N. Klöting and D. Schleinitz contributed equally to this study.

N. Klöting $\cdot$ K. Ruschke $\cdot$ M. Fasshauer $\cdot$ A. Tönjes $\cdot$

M. Stumvoll $\cdot$ M. Blüher $(\bowtie)$

Department of Medicine, University of Leipzig,

Ph.-Rosenthal-Str. 27,

04103 Leipzig, Germany

e-mail: bluma@medizin.uni-leipzig.de

D. Schleinitz $\cdot$ J. Berndt $\cdot$ P. Kovacs

Junior Research Group N06, Interdisciplinary Center of Clinical

Research Leipzig, Faculty of Medicine, University of Leipzig,

Leipzig, Germany

M. R. Schön

Department of Surgery II, University of Leipzig,

Leipzig, Germany

M. R. Schön

Städtisches Klinikum Karlsruhe, Klinik für Viszeralchirurgie,

Karlsruhe, Germany
Results FTO mRNA expression was threefold higher in subcutaneous than in visceral adipose tissue. Subcutaneous FTO expression correlated with visceral FTO expression. FTO gene expression in both depots correlated with age and was negatively correlated to BMI and per cent body fat. FTO mRNA levels were not related to measures of insulin sensitivity and glucose metabolism. RPGRIPIL mRNA expression was 1.6-fold higher in visceral than in subcutaneous adipose tissue, but did not correlate with anthropometric and metabolic characteristics. There was no association between rs8050136 and FTO or RPGRIPIL mRNA expression in adipose tissue.

Conclusions/interpretation Expression of adipose tissue FTO mRNA is fat depot-specific and negatively correlates with measures of obesity. However, the direction of this relationship still needs to be elucidated.

Keywords Adipose tissue - Fasting plasma glucose $\cdot$ FTO . Genetics of obesity . Glucose metabolism . Insulin sensitivity · Obesity $\cdot R P G R I P 1 L \cdot$ Visceral fat

\author{
Abbreviations \\ FTO fat mass and obesity associated \\ LD linkage disequilibrium
}

\section{Introduction}

Genetic factors play an important role in the development of obesity [1], but the underlying genetic causes are poorly understood [2]. Two independent studies using different approaches recently demonstrated a strong association between variants in the gene fat mass and obesity associated $(F T O)$ and obesity [3, 4]. Frayling et al. [3] found the asso- 
ciation between FTO variants (represented by rs9939609) and obesity in a genome-wide scan for type 2 diabetes and replicated it in a total of more than 38,000 individuals. The association between SNPs in the FTO gene and type 2 diabetes was shown to be due to obesity [3]. In agreement with these findings, Dina et al. [4] reported a set of SNPs in the FTO gene (rs1421085 and rs17817449) that is consistently strongly associated with early-onset childhood and severe adult obesity. Both groups replicated the association between FTO and obesity in large cohorts [3, 4]. Moreover, recently Scuteri et al. [5] replicated the significant association between rs 9930506 in the FTO gene and BMI, body weight and hip circumference in European and Hispanic Americans.

The Fto gene was originally described in a mouse with fused toes (Ft mutation) [6, 7]. Homozygous $F t$ mutation is embryonic lethal [6]; heterozygous mice have fused toes and hyperplasia of the thymus most likely due to impaired apoptosis [7]. Interestingly, obesity has not been reported in these mice. However, the Ft mutation consists of at least six deleted genes and no conclusions can be drawn from this model regarding the function of FTO and the relationship between the Fto gene and obesity [2]. Human de novo duplication on chromosome 16, which includes the FTO locus, has been shown to be associated with mental retardation, obesity, dysmorphic facies and severe bilateral clinodactyly of some fingers [8]. Recent bioinformatics analysis revealed that FTO shares sequence motifs with $\mathrm{Fe}^{2+}$ - and 2-oxoglutarate-dependent oxygenases [9]. It has also been shown that FTO localises to the nucleus and that recombinant murine FTO catalyses the demethylation of 3-methylthymine in single-stranded DNA [9].

After the association with human obesity was discovered, the name was changed to 'fat mass and obesity associated' (FTO) [2], but the function of the FTO gene is still unknown. FTO gene is expressed in most tissues, including adipose tissue, beta cells and brain [3]. The highest FTO expression levels have been detected in brain $[3,9]$.

Functional studies are necessary to define the biological role of FTO and the major FTO-'sensitive' organs. We therefore tested the hypothesis that FTO mRNA expression in visceral and subcutaneous adipose tissue is linked to obesity in a cohort of individuals with wide ranges of obesity and body fat distribution. In addition to FTO, retinitis pigmentosa GTPase regulator interacting protein 1-like (RPGRIPIL, also known as KIAA1005) is also thought to be involved in the development of obesity because of its genomic position near the FTO gene $[3,5]$. The transcription start site of RPGRIPIL is situated only 200 base pairs from the $5^{\prime}$ end of $F T O$ and $\sim 61 \mathrm{~kb}$ from the $47 \mathrm{~kb}$ interval containing the BMI associations. RPGRIP1L is ubiquitously expressed with relatively high levels in hypothalamus and islet. We therefore also examined RPGRIPIL mRNA expression in visceral and subcutaneous adipose tissue and its relation to obesity.

In addition, we investigated whether previously published obesity-associated FTO variants (represented by rs 8050136 in our study) might explain potential variability in FTO and RPGRIP1L mRNA expression.

\section{Methods}

Participants and measurement of participant characteristics Paired samples of visceral and subcutaneous adipose tissue were obtained from 55 Europid men $(n=26)$ and women $(n=29)$ who underwent open abdominal surgery for elective cholecystectomy or explorative laparotomy. Using OGTTs, we identified individuals with type 2 diabetes $(n=19)$ or normal glucose tolerance $(n=36)$. All participants had stable weight with no fluctuations of $>2 \%$ of body weight for at least 3 months before surgery. Patients with malignant diseases or any acute or chronic inflammatory disease as determined by a leucocyte count $>7,000 \times$ $10^{9} / 1$, C-reactive protein $>50 \mathrm{mg} / 1$ or clinical signs of infection were excluded from the study. Samples of visceral and subcutaneous adipose tissue were immediately frozen in liquid nitrogen after explantation. The study was approved by the ethics committee of the University of Leipzig. All participants gave written informed consent before taking part in the study.

Assays and measures of glucose metabolism and body fat content Basal blood samples were taken after an overnight fast. Plasma insulin was measured with an enzyme immunometric assay for an automated analyser (Immulite; Diagnostic Products, Los Angeles, CA, USA). Plasma leptin levels were assessed by radioimmunoassay (Linco Research, St Charles, MO, USA). OGTT was performed after an overnight fast with $75 \mathrm{~g}$ standardised glucose solution (Glucodex Solution 75 g; Merieux, Montreal, QC, Canada). Insulin sensitivity was assessed with the euglycaemic-hyperinsulinaemic clamp method [10]. Percentage body fat was measured by dualenergy X-ray absorptiometry. In addition, abdominal visceral and subcutaneous fat area was calculated as previously described [11, 12].

Analysis of human FTO and RPGRIPIL mRNA expression Human FTO and RPGRIPIL mRNA expression was measured by quantitative real-time RT-PCR in a fluorescent temperature cycler using the TaqMan assay; fluorescence was detected on an ABI PRISM 7000 sequence detector (Applied Biosystems, Darmstadt, Germany). Total RNA was isolated from paired subcutaneous and visceral adipose tissue samples using TRIzol (Life Technologies, Grand Island, NY, USA), and $1 \mu \mathrm{g}$ RNA was reverse transcribed 
with standard reagents (Life Technologies). From each RTPCR, $2 \mu 1$ was amplified in a $26 \mu \mathrm{l}$ PCR using a kit (Brilliant SYBR Green QPCR Core Reagent Kit; Stratagene, La Jolla, CA, USA) according to the manufacturer's instructions. Samples were incubated in the sequence detector for an initial denaturation at $95^{\circ} \mathrm{C}$ for $10 \mathrm{~min}$, followed by $40 \mathrm{PCR}$ cycles, each cycle consisting of $95^{\circ} \mathrm{C}$ for $15 \mathrm{~s}, 60^{\circ} \mathrm{C}$ for $1 \mathrm{~min}$ and $72^{\circ} \mathrm{C}$ for $1 \mathrm{~min}$. The following primers were used: human FTO 5'-CTGGCCAGTGAAAGGGTCTAAT-3' (sense) and 5'-GGCAGCAAGTTCTTCCAAAGC-3' (antisense); human RPGRIP1L 5'-GGTATTGGCAAGCTCAGGGTAA-3' (sense) and 5'-TCAAGCCTCCAAGTCATCTCTGT-3' (antisense). SYBR Green I fluorescence emissions were monitored after each cycle. Human FTO and RPGRIPIL mRNA expression was calculated relative to the mRNA expression of $18 \mathrm{~S}$ rRNA, determined by a premixed assay on demand for human 18S rRNA (Applied Biosystems, Darmstadt, Germany). Amplification of specific transcripts was confirmed by melting curve profiles (cooling the sample to $68^{\circ} \mathrm{C}$ and heating slowly to $95^{\circ} \mathrm{C}$ with measurement of fluorescence) at the end of each PCR. The specificity of the PCR was further verified by subjecting the amplification products to agarose gel electrophoresis.

Genotyping of the rs8050136 FTO variant The SNP rs8050136 was selected for genotyping as a proxy for SNPs belonging to one linkage disequilibrium (LD) group including the previously reported rs9939609 [3]. The choice of rs8050136 was based on the better quality of genotyping compared with rs9939609. Genotyping of rs8050136 was performed using the TaqMan allelic discrimination assay (Applied Biosystems) on an ABI PRISM 7500 sequence detector (Applied Biosystems). To assess genotyping reproducibility, a random $\sim 10 \%$ selection of the samples was re-genotyped in all SNPs with $100 \%$ concordance. Genotype data were consistent with Hardy-Weinberg equilibrium $(p>0.05)$.

Statistical analyses Data are shown as means \pm SD unless stated otherwise. Before statistical analysis, non-normally distributed parameters were logarithmically transformed to approximate a normal distribution. Expression differences between visceral and subcutaneous adipose tissue were assessed using the paired Student's $t$ test. Linear relationships were assessed by least square regression analysis. Multivariate linear relationships were assessed by a general linear model. Associations between rs8050136 and FTO mRNA expression levels were assessed using generalised linear regression models under the additive and dominant/ recessive mode of inheritance. Statistical analysis was performed using SPSS version 12.0 (SPSS, Chicago, IL, USA). We considered $p$ values $<0.05$ to be statistically significant.

\section{Results}

Participant characteristics The study population comprised 55 Europid volunteers (26 men, 29 women) and was characterised by a mean age of $61 \pm 15$ years, mean BMI of $34.3 \pm 9.5 \mathrm{~kg} / \mathrm{m}^{2}$ (women: $37.7 \pm 10.7 \mathrm{~kg} / \mathrm{m}^{2}$; men: $\left.30.4 \pm 6.1 \mathrm{~kg} / \mathrm{m}^{2}, p<0.05\right)$, mean waist circumference of $119 \pm 26 \mathrm{~cm}$ (women: $121 \pm 28 \mathrm{~cm}$; men: $115 \pm 23 \mathrm{~cm}$ ) and mean body fat mass of $36 \pm 11 \%$ (women: $40.6 \pm 11.1 \%$; men: $30.8 \pm 8.4 \%, p<0.05$ ). Analysis of 55 paired samples of visceral and subcutaneous adipose tissue revealed threefold higher FTO mRNA levels in subcutaneous than in visceral adipose tissue, while RPGRIP1L mRNA expression was 1.6-fold higher in visceral than in subcutaneous adipose tissue (Fig. 1a,b). FTO and RPGRIP1L mRNA gene expression was not different between men and women in either fat depot. Moreover, subcutaneous and visceral FTO mRNA expression were significantly correlated (Fig. 1c).

Associations of FTO expression Visceral FTO mRNA was significantly correlated with BMI (Fig. 1d), per cent body fat, sex and age, but not with visceral fat area (Table 1). Univariate regression analyses revealed further significant correlations between subcutaneous FTO mRNA expression and BMI (Fig. 1e), per cent body fat, subcutaneous fat area, age, sex and leptin (Table 1). The relationship between FTO mRNA expression and BMI was significant independently of sex. However, in our study population the most obese participants were also the youngest and upon statistical adjustment for BMI, the age relationship disappeared (Table 2). Since the univariate correlation with BMI was much stronger, the age effect was most probably secondary to BMI. In a stepwise regression analysis age did not enter the model after BMI at a $p$ level of 0.05 . There was no correlation between leptin and adiponectin serum concentrations, and visceral or subcutaneous FTO mRNA expression beyond the associations of these adipokines with BMI or per cent body fat.

The association between FTO expression and BMI remained significant upon adjustment for age and sex in the visceral $(p=0.003)$, but not in the subcutaneous depot $(p=0.08$; Table 2). Since no functional information is available on how FTO might contribute to human obesity, we also modelled the data by reverse logic, i.e. BMI as dependent variable (Table 3). Interestingly, visceral FTO mRNA expression remained an independent predictor of BMI even after adjustment for subcutaneous FTO mRNA expression.

There were no significant relationships between both visceral and subcutaneous FTO expression and fasting plasma glucose concentration, $\mathrm{HbA}_{1 \mathrm{c}}$ and parameters of insulin sensitivity, including fasting plasma insulin concen- 

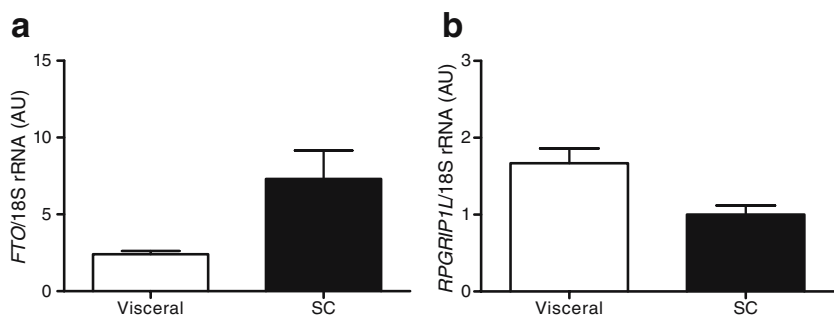

C

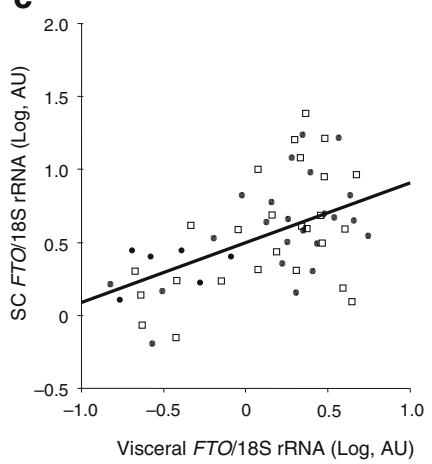

d

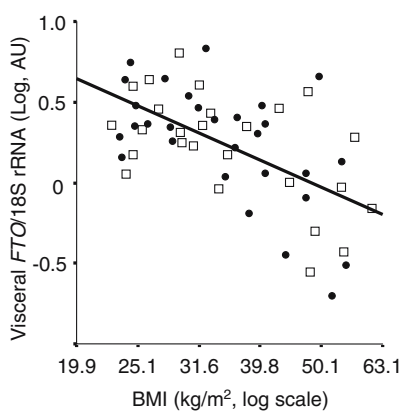

e

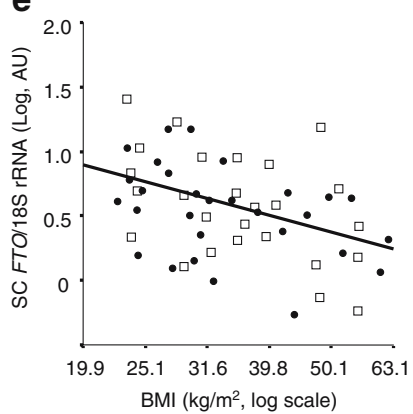

Fig. 1 FTO mRNA and RPGRIPIL mRNA expression in individuals with wide ranges of obesity, body fat distribution, insulin sensitivity and glucose tolerance. Men, white bars/symbols, $n=26$; women, black bars/symbols, $n=29$. a FTO mRNA and b RPGRIPIL mRNA expression in visceral and subcutaneous (SC) adipose tissue. Values are means \pm SEM. $p=0.01$. c Correlation between visceral and SC FTO mRNA expression $(n=55) . p=0.016 ; r^{2}=0.11$. d Correlations between visceral and (e) SC FTO mRNA expression and BMI. $p<0.001 ; r^{2}=$ 0.27 (d); $p=0.018 ; r^{2}=0.11$ (e). Human FTO and RPGRIPIL mRNA expression was calculated relative to the mRNA expression of $18 \mathrm{~S}$ rRNA. Data were log transformed to achieve normal distribution. AU, arbitrary units

trations and glucose uptake during the steady state of a euglycaemic-hyperinsulinaemic clamp (Table 1).

In contrast to FTO, no correlation of RPGRIP $1 L$ mRNA expression with anthropometric and metabolic parameters was seen in either fat depot (Table 1).

Adipose tissue FTO mRNA expression in function of rs8050136 The SNP rs8050136 was selected for genotyping as a proxy for SNPs belonging to one LD group based on HapMap Rel 21a/phaseII (http://www.hapmap.org/cgiperl/gbrowse/hapmap B36/). This LD group includes rs9939609, which is a common variant previously shown to be strongly associated with obesity [3]. No significant association of rs8050136 with visceral or subcutaneous FTO mRNA levels was found (Table 4). However, despite the lack of statistical power due to the small sample size, rs8050136 was still associated with obesity in our study group (data not shown).

\section{Discussion}

Two independent studies recently revealed an association between common variants in the FTO gene on chromosome $16 \mathrm{q} 12.2$, which have been confirmed in more than 20 independent cohorts [3-5], and BMI. FTO is highly expressed in brain, but also detectable in most tissues, including adipose tissue and pancreatic beta cells [3, 9]. The function of the FTO gene product and its potential role for human obesity, however, are completely unknown at the moment. It is possible that only central mechanisms related to the control of appetite and satiety are involved. On the other hand, adipose tissue represents a primary candidate for studying the functional relevance of FTO in obesity. Conceivably, expression differences between lean and obese individuals could contribute to our understanding of the FTO gene product. Based on its genomic position near FTO, as well as on similar expression profiles, RPGRIPIL is also considered a plausible candidate gene involved in the pathophysiology of obesity [3, 5].

In this study, we provide first descriptive information on the relationship between FTO and RPGRIPIL mRNA expression in adipose tissue and simple anthropometric parameters including measures of obesity.

FTO mRNA expression was threefold higher in subcutaneous than in visceral fat. This difference could be due to a number of factors usually differentiating these two depots including gene transcription, insulin sensitivity and lipolysis, altered adipokine expression and others $[13,14]$. Decreasing subcutaneous fat mass does not significantly improve obesityassociated metabolic abnormalities [15], whereas reduction of visceral fat mass has been shown to have significant beneficial effects on glucose metabolism and insulin sensitivity [16]. Our data indicate that fat depot differences in FTO expression may contribute to the specific properties of visceral adipose tissue. The observation that FTO expression in the two depots was only modestly correlated suggests that in a given individual up- or downregulation in one vs the other depot is controlled locally rather than in a coordinated fashion by systemic hormonal, neuronal and/or nutritional signals.

Independently of the fat depot, we found significant negative correlations between FTO gene expression and BMI or per cent body fat. Therefore our data suggest that FTO gene expression might be downregulated in response 
Table 1 Univariate linear regression between anthropometric and biochemical parameters and visceral and subcutaneous FTO mRNA and RPGRIPIL mRNA expression

\begin{tabular}{|c|c|c|c|c|c|c|c|c|}
\hline \multirow[t]{3}{*}{ Parameter } & \multicolumn{4}{|c|}{$F T O / 18 \mathrm{~S}$ rRNA } & \multicolumn{4}{|c|}{ RPGRIPIL/18S rRNA } \\
\hline & \multicolumn{2}{|c|}{ Visceral } & \multicolumn{2}{|c|}{ Subcutaneous } & \multicolumn{2}{|c|}{ Visceral } & \multicolumn{2}{|c|}{ Subcutaneous } \\
\hline & $r$ & $p$ value & $r$ & $p$ value & $r$ & $p$ value & $r$ & $p$ value \\
\hline Age & 0.55 & $<0.001$ & 0.32 & 0.03 & 0.04 & 0.79 & 0.05 & 0.76 \\
\hline Sex & -0.33 & 0.02 & -0.45 & 0.01 & -0.03 & 0.87 & 0.23 & 0.15 \\
\hline Visceral fat area & -0.08 & 0.64 & -0.18 & 0.27 & 0.19 & 0.43 & -0.13 & 0.57 \\
\hline Subcutaneous fat area & -0.06 & 0.7 & -0.31 & 0.04 & 0.21 & 0.37 & -0.10 & 0.67 \\
\hline Per cent body fat & -0.3 & 0.03 & -0.41 & 0.003 & 0.16 & 0.34 & -0.04 & 0.79 \\
\hline Waist circumference & -0.2 & 0.2 & -0.28 & 0.06 & 0.35 & 0.06 & 0.03 & 0.87 \\
\hline FPG & -0.27 & 0.07 & -0.19 & 0.2 & 0.23 & 0.16 & -0.09 & 0.57 \\
\hline $\mathrm{HbA}_{1 \mathrm{c}}$ & -0.17 & 0.25 & -0.21 & 0.15 & 0.06 & 0.71 & -0.29 & 0.07 \\
\hline Glucose uptake, clamp & -0.02 & 0.9 & 0.05 & 0.8 & -0.19 & 0.33 & -0.12 & 0.54 \\
\hline FPI & -0.06 & 0.7 & -0.04 & 0.8 & 0.24 & 0.14 & -0.08 & 0.63 \\
\hline Leptin & -0.29 & 0.04 & -0.31 & 0.04 & 0.17 & 0.35 & 0.23 & 0.20 \\
\hline
\end{tabular}

Participants: $n=55$

FPG, fasting plasma glucose; FPI, fasting plasma insulin

to fat accumulation. As indicated by the models (Table 2), this inverse relationship was significantly stronger for the visceral depot. From our correlation analysis it is impossible to extricate the direction of causality. Nevertheless, since genetic variation in FTO has been shown to affect the risk of obesity, it is legitimate to test the hypothesis of whether FTO expression predicts BMI. We identified visceral FTO mRNA expression as a strong predictor of BMI independently of age and sex, and even independently of FTO mRNA expression in the subcutaneous depot. This suggests that especially viscerally expressed FTO may contribute to obesity.

Obviously, the number of participants in our study was relatively small and the association between BMI and adipose tissue FTO mRNA expression levels will require

Table 2 Multiple regression analysis between age, sex, BMI and FTO mRNA expression in visceral and subcutaneous adipose tissue

\begin{tabular}{|c|c|c|}
\hline & \multicolumn{2}{|c|}{$\beta$-Coefficient ( $p$ value) } \\
\hline & $\begin{array}{l}(\log ) \text { Visceral FTO } \\
\text { mRNA }\end{array}$ & $\begin{array}{l}\text { (log) Subcutaneous } \\
\text { FTO mRNA }\end{array}$ \\
\hline \multicolumn{3}{|l|}{ Model 1} \\
\hline Age (log) & $0.49(0.003)$ & $0.17(0.39)$ \\
\hline Sex (female) & $-0.08(0.08)$ & $-0.06(0.36)$ \\
\hline \multicolumn{3}{|l|}{ Model 2} \\
\hline Age (log) & $0.26(0.1)$ & $-0.003(0.99)$ \\
\hline BMI $(\log )$ & $-0.66(0.0006)$ & $-0.52(0.049)$ \\
\hline \multicolumn{3}{|l|}{ Model 3} \\
\hline Age $(\log )$ & $0.27(0.09)$ & $0.002(0.99)$ \\
\hline Sex (female) & $-0.02(0.59)$ & $-0.01(0.89)$ \\
\hline BMI (log) & $-0.62(0.003)$ & $-0.51(0.08)$ \\
\hline
\end{tabular}

Participants: $n=55$ confirmation in larger cohorts. We also cannot exclude the possibility that specific characteristics of our study population, i.e. the strong negative correlation between age and BMI, may have produced a spurious relationship between FTO gene expression and obesity parameters. However, the fact that the BMI association withstood all conceivable adjustment makes us confident that this finding is robust. Adipose FTO expression and obesity could be coregulated by a common genetic or environmental factor and not functionally linked to each other. More sophisticated study designs are necessary to dissect causal from correlative relationships with FTO expression in fat.

Table 3 Multivariate linear regression analysis of visceral and subcutaneous FTO mRNA expression as predictor of BMI

\begin{tabular}{|c|c|c|}
\hline & \multicolumn{2}{|l|}{ BMI } \\
\hline & $\beta$-Coefficient & $p$ value \\
\hline \multicolumn{3}{|l|}{ Model 1} \\
\hline Age (log) & -0.32 & 0.003 \\
\hline Sex (female) & 0.09 & 0.008 \\
\hline SC FTO $(\log )$ & -0.12 & 0.08 \\
\hline \multicolumn{3}{|l|}{ Model 2} \\
\hline Age $(\log )$ & -0.20 & 0.06 \\
\hline Sex (female) & 0.07 & 0.02 \\
\hline Visceral FTO $(\log )$ & -0.29 & 0.003 \\
\hline \multicolumn{3}{|l|}{ Model 3} \\
\hline Age (log) & -0.20 & 0.056 \\
\hline Sex (female) & 0.07 & 0.03 \\
\hline Visceral FTO (log) & -0.26 & 0.01 \\
\hline SC FTO $(\log )$ & -0.07 & 0.31 \\
\hline
\end{tabular}

Participants: $n=55$

$\mathrm{SC}$, subcutaneous 
Table 4 FTO and RPGRIPIL mRNA expression in visceral and subcutaneous adipose tissue grouped by rs 8050136 genotypes

\begin{tabular}{|c|c|c|c|c|c|c|c|c|c|}
\hline \multirow[t]{2}{*}{ Genotype rs8050136 } & \multirow[t]{2}{*}{$n$} & \multicolumn{4}{|c|}{ FTO mRNA expression (AU) } & \multicolumn{4}{|c|}{ RPGRIP1L mRNA expression (AU) } \\
\hline & & Visceral & $p$ value & $\mathrm{SC}$ & $p$ value & Visceral & $p$ value & $\mathrm{SC}$ & $p$ value \\
\hline $\mathrm{CC}$ & 13 & $2.63 \pm 0.39$ & 0.72 (add) & $6.44 \pm 1.41$ & 0.90 (add) & $1.80 \pm 0.64$ & 0.74 (add) & $1.11 \pm 0.33$ & 0.42 (add) \\
\hline CA & 24 & $2.49 \pm 0.34$ & 0.60 (dom) & $8.41 \pm 3.79$ & 0.73 (dom) & $1.81 \pm 0.26$ & 0.91 (dom) & $1.00 \pm 0.18$ & 0.64 (dom) \\
\hline AA & 11 & $2.22 \pm 0.41$ & $0.96(\mathrm{rec})$ & $6.90 \pm 1.72$ & $0.56(\mathrm{rec})$ & $1.73 \pm 0.27$ & $0.69(\mathrm{rec})$ & $1.03 \pm 0.19$ & $0.41(\mathrm{rec})$ \\
\hline
\end{tabular}

Data are means \pm SEM

Generalised linear models were used to identify significant differences between genotypes under the additive (add), dominant (dom) and recessive (rec) models, after adjustment for age, sex and BMI

AU, arbitrary units; SC, subcutaneous

We did not find an association between FTO mRNA levels and either fasting plasma glucose concentrations, $\mathrm{HbA}_{1 \mathrm{c}}$ or parameters of insulin sensitivity. However, we cannot rule out the possibility that our sample size was too small to detect significant relationships between FTO gene expression and measures of insulin sensitivity and glucose metabolism. At the least, our data suggest that adipose tissue FTO expression does not directly affect risk of type 2 diabetes. As such, our results are generally supportive of the findings by Frayling et al. [3], who reported that the original association between FTO SNPs and type 2 diabetes disappeared upon adjustment for BMI.

Although expression of RPGRIPIL mRNA was also fat depot-specific in our study, it does not, in contrast to FTO, seem to be linked to the pathophysiology of obesity as no correlations with anthropometric and metabolic characteristics were found. Our data therefore favour FTO rather than RPGRIPIL as a possible target affected by reported obesity-associated polymorphisms [3]. Recently, mutations within RPGRIPIL have been shown to be responsible for several syndromes such as Joubert or Meckel syndrome $[17,18]$. Joubert syndrome is an autosomal recessive multisystem disease characterised by cerebellar ataxia, developmental delay, hypotonia, irregular breathing pattern, eye movement abnormalities and cerebellar vermis hypoplasia and dysplasia with accompanying brainstem defects [18]. Meckel syndrome is an autosomal recessive lethal condition characterised by central nervous system malformation, postaxial polydactyly, cystic kidney dysplasia and ductal proliferation in the portal area of the liver $[17,18]$. No obesity phenotype has been reported for these syndromes caused by RPGRIPIL mutations.

Given the strength of the correlation between FTO expression and adiposity in the present study and the relatively small effect that variation in FTO has on BMI as reported by Frayling et al. [3], variation in the FTO genotype does not appear to play a major role in regulating FTO transcription in adipose tissue. This is supported by the fact that no association between the obesity risk polymorphism and FTO and/or RPGRIPIL mRNA expression in adipose tissue was found in our study. It is noteworthy that, despite our study's lack of statistical power due to the small sample size, rs8050136 was still associated with obesity in this study group.

In conclusion, expression of adipose tissue FTO mRNA is fat depot-specific and correlates with age, while being negatively correlated with BMI and total body fat. Adipose tissue FTO mRNA expression is not related to rs8050136 genotype, which is in LD with the previously reported obesity-associated SNP rs9939609 [3]. However, we were unable to determine the causal direction of the association between obesity and alterations in FTO gene expression. Therefore further studies are necessary to elucidate whether changes in adipose tissue FTO gene expression are secondary to obesity or causally related.

Acknowledgements This work was supported by grants from: (1) Deutsche Forschungsgemeinschaft (DFG) BL 580/3-1 (M. Blüher); (2) the Clinical Research group 'Atherobesity' KFO 152, projects BL 833/1-1 (M. Blüher), Stu192/6-1 (M. Stumvoll) and FA 476/4-1 (M. Fasshauer); and (3), the Interdisciplinary Center of Clinical Research (IZKF) Leipzig at the Faculty of Medicine of the University of Leipzig (Project N06 to J. Berndt, D. Schleinitz and P. Kovacs, Project B24 to M. Blüher and Project B27 to M. Stumvoll).

Duality of interest The authors declare that there is no duality of interest associated with this manuscript.

\section{References}

1. Maes HH, Neale MC, Eaves LJ (1997) Genetic and environmental factors in relative body weight and human adiposity. Behav Genet $27: 325-351$

2. Groop L (2007) From fused toes in mice to human obesity. Nat Genet 39:706-707

3. Frayling TM, Timpson NJ, Weedon MN et al (2007) A common variant in the FTO gene is associated with body mass index and predisposes to childhood and adult obesity. Science 316:889-894

4. Dina C, Meyre D, Gallina S et al (2007) Variation in FTO contributes to childhood obesity and severe adult obesity. Nat Genet 39:724-726

5. Scuteri A, Sanna S, Chen WM et al (2007) Genome-wide association scan shows genetic variants in the FTO gene are associated with obesity-related traits. PLoS Genet 3:e115 
6. van der Hoeven F, Schimmang T, Volkmann A, Mattei MG, Kyewski B, Ruther U (1994) Programmed cell death is affected in the novel mouse mutant Fused toes (Ft). Development 120:2601-2607

7. Peters T, Ausmeier K, Ruther U (1999) Cloning of Fatso (Fto), a novel gene deleted by the Fused toes $(\mathrm{Ft})$ mouse mutation. Mamm Genome 10:983-986

8. Stratakis CA, Lafferty A, Taymans SE, Gafni RI, Meck JM, Blancato J (2000) Anisomastia associated with interstitial duplication of chromosome 16, mental retardation, obesity, dysmorphic facies, and digital anomalies: molecular mapping of a new syndrome by fluorescent in situ hybridization and microsatellites to 16q13 (D16S419-D16S503). J Clin Endocrinol Metab 85: 3396-3401

9. Gerken T, Girard CA, Tung YC et al (2007) The obesityassociated FTO gene encodes a 2-oxoglutarate-dependent nucleic acid demethylase. Science 318:1469-1472

10. DeFronzo RA, Tobin JD, Andres R (1979) Glucose clamp technique: a method for quantifying insulin secretion and resistance. Am J Physiol 237:214-223

11. Berndt J, Klöting N, Kralisch S et al (2005) Plasma visfatin concentrations and fat depot-specific mRNA expression in humans. Diabetes 54:2911-2916
12. Klöting N, Graham TE, Berndt J et al (2007) Serum retinol-binding protein is more highly expressed in visceral than in subcutaneous adipose tissue and is a marker of intra-abdominal fat mass. Cell Metab 6:79-87

13. Wajchenberg BL (2000) Subcutaneous and visceral adipose tissue: their relation to the metabolic syndrome. Endocr Rev 21:697-738

14. Frayn KN (2000) Visceral fat and insulin resistance-causative or correlative? Br J Nutr 83:S71-S77

15. Klein S, Fontana L, Young VL et al (2004) Absence of an effect of liposuction on insulin action and risk factors for coronary heart disease. N Engl J Med 350:2549-2557

16. Thorne A, Lonnqvist F, Apelman J, Hellers G, Arner P (2002) A pilot study of long-term effects of a novel obesity treatment: omentectomy in connection with adjustable gastric banding. Int J Obes Relat Metab Disord 26:193-199

17. Delous M, Baala L, Salomon R et al (2007) The ciliary gene RPGRIP1L is mutated in cerebello-oculo-renal syndrome (Joubert syndrome type B) and Meckel syndrome. Nat Genet 39: 875-881

18. Arts HH, Doherty D, van Beersum SEC et al (2007) Mutations in the gene encoding the basal body protein RPGRIP1L, a nephrocystin-4 interactor, cause Joubert syndrome. Nat Genet $39: 882-888$ 